\title{
Evaluation of Different Brain Imaging Technologies
}

\author{
Leqi Tong
}

\author{
Institution of education \\ University College London \\ Corresponding author.Email: dtnvlto@ucl.ac.uk
}

\begin{abstract}
To detect disorders caused by various brain regions impaired, many neuroimaging techniques are routinely utilized, such as electroencephalography (EEG) and functional magnetic resonance imaging (fMRI) that may be the most common imaging techniques used to detect. This article summarizes and discusses the benefits and drawbacks of different neuroimaging techniques, as well as the benefits and limitations of some combined imaging techniques to have a clearer perception of neuroimaging technologies. In addition, this paper will also specifically focus on fNIRS and EGG-MEG combining techniques. The result shows that EEG has a high temporal resolution and fMRI has a high spatial resolution, so EEG and fMRI are generally combined to diagnosis particular disorders. A key point is that the combined use of EEG and fMRI may lead to inaccurate results. The EGG-MEG provides a new direction for the continuous treatment of epilepsy or other brain diseases.
\end{abstract}

Keywords: neuroimaging techniques, EEG, fMRI, fNIRS, advantages

\section{INTRODUCTION}

Many functional neuroimaging techniques, such as electroencephalography (EEG), functional magnetic resonance imaging (fMRI), functional near-infrared spectroscopy (fNIRS), and magnetoencephalography (MEG), have been used to detect some brain lesions by providing information about the activity of neurons during sensory or cognitive tasks. The diverse neuroimaging techniques are mainly geared at detecting distinct brain injuries depending on the imaging mechanism of the different neuroimaging techniques. For example, EEG is generally suitable for diagnosing some aberrant brain processes such as ADHD, children with attention deficit hyperactivity disorder (ADHD) exhibit EEG abnormalities including excess slow brainwaves and epileptiform spike and wave activity [1]. In terms of functional magnetic resonance imaging (fMRI), autism spectrum disorders (ASD) can be detected by it, which uses differences in the ferromagnetic properties of oxygenated and deoxygenated blood to generate an indirect measure of neuronal activity that can be used to analyze sensory processing, executive function, and mental states in ASD patients [2]. Moreover, concerning functional Near Infrared spectroscopy (fNIRS), it has been significant in the study of neonatal brain hemodynamics, and dual-wavelength fNIRS can also be used to detect critically low oxygen levels, resulting in increased cerebral oxygen saturation during acute heart failure treatment [3]. For MEG, Blast-induced mild traumatic brain injury (TBI) can be detected by it, which can detect and localize the aberrant low-frequency magnetic activity in the affected brain tissue [4]. However, each imaging technique has its own set of benefits and limits, it is usually necessary to combine two complementary techniques to evaluate certain illnesses, such as epilepsy, in order to overcome the deficiencies of one technique. There are many researches and discussions on the advantages and disadvantages of a single technology, but there are relatively few discussions and analysis on the combination of the two technologies. Therefore, this paper aims to analyze and outline the advantages and disadvantages of different neuroimaging techniques, as well as to explore the advantages and limitations of some combining neuroimaging techniques: combining EEG and fMRI; combining MEG and EEG. This article can not only provide a research basis for the understanding of neuroimaging technology, but also provide a constructive thinking for the future technological progress.

\section{ADVANTAGES AND LIMITATIONS OF EEG}

Electroencephalography (EEG) is a neuroimaging technology that records brain activity and diagnoses 
various neural illnesses by measuring electrical impulses generated by electrodes placed on the scalp [5]. The most important advantage of EEG is its extraordinarily high temporal resolution, which allows electrical impulses to be recorded thousands of times per second [6]. For example, some disorders like epilepsy propagate rapidly, dealing with this problem necessitates an approach with a high temporal resolution. Therefore, EEG is recognized as the best non-invasive diagnosis tool utilized in epilepsy centers [5]. On the other hand, EEG is also suitable for investigating cognitive development, particularly examining the changes in brain development throughout infancy and early childhood [7], because of its high temporal resolution, postsynaptic alterations can be immediately reflected in the EEG, which may be beneficial for tracking rapid changes in brain functioning of younger participants [7]. Although EEG is one of the better brain imaging tools for detecting rapidly changing brain activities, it has one major drawback that it has a low spatial resolution, making it difficult for researchers to accurately localize activity to motor or sensory areas, or to differentiate between different portions of the primary motor area [6]. EEG generally uses scalp electrodes to record brain electrical activity that occurs several centimeters below the recording electrodes [8]. As a result, cortical current should pass through a variety of resistant layers. EEG scans may cause spatial blurring due to the varying conductivities of the cortex, dura mater, skull, and scalp [9]. As a result, this volume-conductioninduced mixing could be the root cause of scalp EEG's poor spatial resolution [8].

\section{ADVANTAGES AND LIMITATIONS OF FMRI}

Functional Magnetic Resonance Imaging(fMRI) is a non-invasive technique for imaging BOLD signal fluctuations that helps researchers to detect the activated brain regions for particular stimuli or various cognitive task processing [10]. Compared to other imaging techniques, fMRI has several distinct benefits. The first one is that it has a high spatial resolution in the millimeter and potentially submillimeter range [11]. This allows for more exact spatial mapping of brain responses, such as detecting finger and digit representations in the somatosensory cortex, monitoring the fine-grained architecture of subcortical structures, and even detecting animal subcortical activity [12]. The second is that it can detect three-dimensional activities deep within the brain. EEG and MEG-based approaches, according to Crosson et al (2010), may have trouble imaging activity in deep, subcortical locations, whereas fMRI can [13]. In addition, with fMRI, the same platform that was used to obtain functional images can also be utilized to acquire highresolution anatomic images in the same space as functional images, allowing for exact anatomic localization. PET, MEG, and EEG, on the other hand, must all rely on structural MR images or atlases to pinpoint activity in the brain [13]. The third one is that fMRI is safer than PET as there are no radioactive contrast agents or metabolites necessary for fMRI. For example, to apply PET as a functional imaging method for cognitive processes, a radiochemist must be nearby, which includes exposing the individual to radiation [13]. fMRI, on the other hand, still has significant drawbacks. The inadequate temporal resolution of $\mathrm{fMRI}$ is its most noticeable flaw. EEG and MEG approaches have better temporal resolution than fMRI. The ability of fMRI to resolve neuronal events is on the order of seconds, compared to milliseconds for EEG and MEG-based approaches [13].

\section{ADVANTAGES AND LIMITATIONS OF FNIRS}

Functional Near-Infrared spectroscopy (fNIRS) may be a neuroimaging technique that measures changes in haemoglobin concentration caused by neuronal activity by using near-infrared light [14]. fNIRS provides many advantages over other sorts of neuroimaging. For instance, the temporal resolution of fNIRS is substantially better than that of fMRI, which suggests brain signals could also be routinely seen with a temporal sampling resolution of 0.01 seconds [15]. This high temporal resolution is especially useful for analyzing brain functional connectivity [16]. As an example, fNIRS systems are often applied to the study of languages like detecting brain activation, cortical lateralization, and functional connectivity between the Broca's and Wernicke's regions [17]. Additionally, compared to EEG, it provides a better spatial resolution of activation images, allowing the localization of brain responses to specific cortical areas [18]. Similarly, Wilcox and Biondi (2015) also mentioned that the effect of fNIRS are often localized within 1-2 $\mathrm{cm}$ of the stimulated area, allowing more precise identification of the cortical response obtained compared to electrophysiological methods [15]. However, fNIRS features a better spatial resolution than EEG, but is inferior to fMRI [19], which can make it difficult to differentiate neural responses in cortical areas [15]. Additionally, thanks to the high level of light scattering within the tissue, fNIRS features a limited penetration depth. As near-infrared light diffuses rapidly in neural tissue, fNIRS is ineffective for detecting neural activation in regions deeper than $1 \mathrm{~cm}$ under the surface of the brain [15]-[16]. Furthermore, fNIRS cannot show specific anatomical information about the brain area, which can make it impossible to spot the brain area from which the signal originated [15]-[16].

\section{ADVANTAGES AND LIMITATIONS OF COMBINING EEG AND FMRI}

Since each neuroimaging technique has its own set of strengths and limitations, combining imaging techniques is going to be critical within the future study of brain 
processes. for instance, EEG-fMRI has been utilized in much research. In terms of temporal and spatial resolution of brain activity, electroencephalography (EEG) and functional magnetic resonance imaging (fMRI) recordings offer complementary advantages. fMRI allows for the precise localization of brain areas involved in cognitive processes, but blood oxygen level-dependent (BOLD) responses are too slow, resulting in inadequate capture of the rich temporal dynamics. EEG signals, on the other hand, are directly associated with the electrical activity of neurons with millisecond accuracy; nonetheless, due to the fuzzy spatial mixture of underlying cortical activity, only limited spatial resolution can be provided by it [20]. the combination of EEG and fMRI could become an important tool within the diagnosis and treatment of epilepsy. As Safi-Harb, et al (2015) mentioned, EEG-fMRI could be a useful technique for epilepsy research. It combines the high temporal resolution of EEG with the high spatial resolution of fMRI, which may localize and detect specific changes in BOLD during a short time [21]. Similarly, Rosenkranz and Lemieux (2010) also stated that EEG-fMRI offers reliable spatial localization of epileptiform in EEG activity as a part of the pre-surgical examination of patients with cortical epilepsy, which is crucial in both the diagnosis and treatment of epilepsy [22]. Therefore, simultaneous recording of EEG and fMRI may provide a big enhancement that helps researchers to possess a deeper understanding of the brain's cognitive function. However, interactions between recording devices may cause signal loss or degradation. For instance, EEG recording electrodes may interfere with MR image acquisition systems because they are made from conductive materials with magnetic properties that differ from those of the surrounding tissue, and MR image acquisition systems are extremely sensitive to magnetic differences within the sector, which can lead to impaired image quality. By contrast, an fMRI scan involves strong static and time-varying electromagnetic fields that can be detected by EEG recording systems, which could trigger the movement of electrodes with the subject and cause artifacts within the EEG signal, especially pulse-related and gradientswitching artifacts [22].

\section{ADVANTAGES AND LIMITATIONS OF COMBINING MEG AND EEG}

In the treatment of epilepsy, combining EEG and MEG is a viable technique. The signals examined by EEG that travel through the weakly conductivity skull, according to Aydin et al (2015), may diminish the electric potentials spatially [23]. The skull conductivity, on the other hand, have a modest to non-existent effect on the magnetic fields detected by the MEG. As a result, EEG may be particularly susceptible to uncertainty and changes in skull conductivities, whereas MEG has been demonstrated to be rather resistant to such changes [23].
Similarly, Stam (2010) also mentioned that, unlike EEG, MEG does not require a reference electrode and is barely influenced by the skull, allowing it to deliver a more precise image of brain activity. In addition, while EEG can detect both tangential and radial components of a current source [24], MEG can only detect tangential components [25], as stated by Bast, et al (2007), MEG signals are primarily produced by intra-neuronal currents from tangentially oriented sources, so MEG may not detect pure radial sources at all [26]. Therefore, MEG selectively measures sulci activity, whereas scalp EEG records both sulci activity and cortical gyri activity. As a result, MEG is more sensitive to superficial cortical activity than to deep sources, making it ideal for neocortical epilepsy research [25]. In conclusion, MEG is unaffected by the skull and scalp, allowing for more precise localization, but it is not affected by radial sources. The skull may attenuate EEG signals, making it less accurate in its localization; but it is sensitive to all source orientations [27], so combining EEG and MEG can be utilized consistently in the treatment of epilepsy or other brain disorders.

\section{DISCUSSION}

The neuroimaging techniques discussed in this paper have their own advantages and limitations. Electroencephalography (EEG) has an extraordinarily high temporal resolution, making it suitable for studying illnesses like epilepsy or cognitive development that involve rapid changes in neuron activity. However, due to its low spatial resolution, proper localization of particular brain processes is difficult to detect.

The first advantage of functional magnetic resonance imaging (fMRI) is that it has a high spatial resolution. The second is that it can detect three-dimensional activities deep within the brain. The third benefit is that fMRI is safe because no radioactive agents are produced. fMRI, on the other hand, has a lower temporal resolution than EEG. For Functional Near Infrared spectroscopy (fNIRS), it has better temporal resolution better than that of fMRI and provides a higher spatial resolution than EEG. fNIRS, on the other hand, has a lower spatial resolution than fMRI. In addition, Because of the high quantity of light scattering within tissue, fNIRS has a restricted penetration depth. Even anatomical information regarding the brain area is not visible with fNIRS.

Due to the limitations of using one particular neuroimaging technique, some combined neuroimaging techniques have been applied, such as EEG-fMRI, which combines the high temporal resolution of EEG with the high spatial resolution of fMRI. In addition, EEG-MEG is also a common combined neuroimaging technique that combines the skull-independent nature of the MEG signal with the sensitivity of EEG to the direction of all signal sources to localize neuronal activity more accurately. 
However, there are still some problems with these techniques, for example recording devices may interfere with each other, the magnetic material of the EEG may interfere with the image acquisition system of the fMRI, and the electromagnetic fields generated during the fMRI scan may cause artefacts in the EEG signal. Therefore, future technical progress point should focus on reducing the impact of interactions between recording techniques, for example by investigating how to eliminate artefacts, or develop new combined techniques to better diagnose brain disorders.

\section{CONCLUSION}

This paper mainly discusses the applicability and limitations of three brain imaging technologies in practical application, that is, EEG, fMRI, fNIRS. Next, the effect of the combination of brain imaging technologies is discussed in depth, that is, EEG and fMRI, MEG and EEG. In conclusion, in the future, single neuroimaging technology for detecting damage caused by brain damage remains the foundation. Due to the limitations of each technology, a broader approach is to combine the respective advantages of two or more technologies. EEG-fMRI is a good application which well combines high temporal resolution and high spatial resolution. EEG-MEG makes good use of independence and sensitivity, and is a very good practice in locating neuronal activity. Through these analyses, this paper can not only provide a research basis for understanding neuroimaging technology, but also provide constructive ideas for the future technological progress.

The limitation of this paper is that there are more theoretical discussions on each technology, and the data collected is relatively small. Future research will more indepth study the data comparative analysis of various technologies in practical application cases, as well as the analysis of practical cases in which various technologies are deeply combined.

\section{ACKNOWLEDGMENTS}

I am sincerely grateful to my professors and teachers who have provided me with invaluable guidance at every stage of the writing of this thesis. They gave me a lot of practical suggestions on the topic selection and specific writing matters. My professor, in particular, patiently pointed out many areas that needed improvement.

\section{REFERENCES}

[1] Loo, S. K., \& Barkley, R. A, Clinical utility of EEG in attention deficit hyperactivity disorder, Applied neuropsychology, vol. 12(2), 2005, pp. 64-76.

[2] Philip, R. C., Dauvermann, M. R., Whalley, H. C., Baynham, K., Lawrie, S. M., \& Stanfield, A. C., A systematic review and meta-analysis of the fMRI investigation of autism spectrum disorders, Neuroscience \& Biobehavioral Reviews, vol. 36(2), 2012, pp. 901-942.

[3] Irani, F., Platek, S. M., Bunce, S., Ruocco, A. C., \& Chute, D., Functional near infrared spectroscopy (fNIRS): an emerging neuroimaging technology with important applications for the study of brain disorders, The Clinical Neuropsychologist, vol. 21(1), 2007, pp. 9-37.

[4] Huang, M., Risling, M., \& Baker, D. G., The role of biomarkers and MEG-based imaging markers in the diagnosis of post-traumatic stress disorder and blastinduced mild traumatic brain injury, Psychoneuroendocrinology, vol. 63, 2016, pp. 398-409.

[5] Jatoi, M. A., Kamel, N., Malik, A. S., Faye, I., \& Begum, T., A survey of methods used for source localization using EEG signals, Biomedical Signal Processing and Control, vol. 11, 2014, pp. 42-52.

[6] Enders, H., \& Nigg, B. M., Measuring human locomotor control using EMG and EEG: Current knowledge, limitations and future considerations, European journal of sport science, vol. 16(4), 2016, pp. 416-426.

[7] Bell, M. A., \& Cuevas, K., Using EEG to study cognitive development: Issues and practices, Journal of cognition and development, vol. 13(3), 2012, pp. 281-294.

[8] Burle, B., Spieser, L., Roger, C., Casini, L., Hasbroucq, T., \& Vidal, F., Spatial and temporal resolutions of EEG: Is it really black and white? A scalp current density view. International Journal of Psychophysiology, vol. 97(3), 2015, pp. 210-220.

[9] Babiloni, F., Cincotti, F., Carducci, F., Rossini, P. M., \& Babiloni, C., Spatial enhancement of EEG data by surface Laplacian estimation: the use of magnetic resonance imaging-based head models, Clinical Neurophysiology, vol. 112(5), 2001, pp. 724-727.

[10] Daliri, M. R., \& Behroozi, M., Advantages and disadvantages of resting state functional connectivity magnetic resonance imaging for clinical applications, OMICS Journal of Radiology, vol. 3(1), 2013, pp. 1-2.

[11] Rodic, S., \& Zhao, P. J. A brief review of neuroimaging using functional magnetic resonance imaging (fMRI), University of Western Ontario Medical Journal, vol. 84(1), 2015, pp. 10-12.

[12] Goense, J., Bohraus, Y., \& Logothetis, N. K., fMRI at high spatial resolution: implications for BOLD- 
models, Frontiers in computational neuroscience, vol. 10, 2016, p. 66.

[13] Crosson, B., Ford, A., McGregor, K. M., Meinzer, M., Cheshkov, S., Li, X., ... \& Briggs, R. W., Functional imaging and related techniques: an introduction for rehabilitation researchers. Journal of rehabilitation research and development, vol. 47(2), 2010, p. vii.

[14] Pinti, P., Aichelburg, C., Lind, F., Power, S., Swingler, E., Merla, A., ... \& Tachtsidis, I., Using fiberless, wearable fNIRS to monitor brain activity in real-world cognitive tasks, Journal of visualized experiments: JoVE, (106), 2012.

[15] Wilcox, T., \& Biondi, M., fNIRS in the developmental sciences, Wiley Interdisciplinary Reviews: Cognitive Science, vol. 6(3), 2015, pp. 263-283.

[16] Tak, S., \& Ye, J. C., Statistical analysis of fNIRS data: a comprehensive review, Neuroimage, vol. 85, 2014, pp. 72-91.

[17] Hall, M., Chaudhary, U., Rey, G., \& Godavarty, A., Fronto-temporal mapping and connectivity using NIRS for language-related paradigms, Journal of Neurolinguistics, vol. 26(1), 2013, pp. 178-194.

[18] Lloyd-Fox, S., Blasi, A., \& Elwell, C. E., Illuminating the developing brain: the past, present and future of functional near infrared spectroscopy, Neuroscience \& Biobehavioral Reviews, vol. 34(3), 2010, pp. 269-284.

[19] Minagawa-Kawai, Y., Mori, K., Hebden, J. C., \& Dupoux, E., Optical imaging of infants' neurocognitive development: recent advances and perspectives, Developmental neurobiology, vol. 68(6), 2008, pp. 712-728.

[20] Debener, S., Ullsperger, M., Siegel, M., \& Engel, A. K., Single-trial EEG-fMRI reveals the dynamics of cognitive function, Trends in cognitive sciences, 10(12), 2006, pp. 558-563.

[21] Safi-Harb, M., Proulx, S., von Ellenrieder, N., \& Gotman, J., Advantages and disadvantages of a fast fMRI sequence in the context of EEG-fMRI investigation of epilepsy patients: a realistic simulation study, Neuroimage, vol. 119, 2015, pp. 20-32.

[22] Rosenkranz, K., \& Lemieux, L., Present and future of simultaneous EEG-fMRI. Magnetic Resonance Materials in Physics, Biology and Medicine, vol. 23(5-6), 2010, pp. 309-316.

[23] Aydin, Ü., Vorwerk, J., Dümpelmann, M., Küpper, P., Kugel, H., Heers, M., ... \& Wolters, C. H,
Combined EEG/MEG can outperform single modality EEG or MEG source reconstruction in presurgical epilepsy diagnosis, PloS one, vol. 10(3), 2015.

[24] Stam, C. J., Use of magnetoencephalography (MEG) to study functional brain networks in neurodegenerative disorders, Journal of the neurological sciences, vol. 289(1-2), 2010, pp. 128134.

[25] Baumgartner, C., Controversies in clinical neurophysiology, MEG is superior to EEG in the localization of interictal epileptiform activity: Con. Clinical neurophysiology, vol. 115(5), 2004, pp. 1010-1020.

[26] Bast, T., Wright, T., Boor, R., Harting, I., Feneberg, R., Rupp, A., ... \& Baumgärtner, U., Combined EEG and MEG analysis of early somatosensory evoked activity in children and adolescents with focal epilepsies, Clinical neurophysiology, vol. 118(8), 2007, pp. 1721-1735.

[27] Ebersole, J. S., \& Ebersole, S. M., Combining MEG and EEG source modeling in epilepsy evaluations, Journal of Clinical Neurophysiology, vol. 27(6), 2010, pp. 360-371. 